\title{
Temperature-dependent refractive index measurements of L-BBH2 glass for the Subaru CHARIS integral field spectrograph
}

\author{
${ }^{\mathrm{a}}$ Douglas B. Leviton, ${ }^{\mathrm{b}}$ Kevin H. Miller, ${ }^{\mathrm{b}}$ Manuel A. Quijada, ${ }^{\mathrm{c}}$ Tyler D. Groff \\ aLeviton Metrology Solutions, Inc., Boulder, CO 80301 \\ bNASA Goddard Space Flight Center, Greenbelt, MD 20771 \\ 'Princeton University, Princeton, NJ 08544
}

\begin{abstract}
Using the Cryogenic High Accuracy Refraction Measuring System (CHARMS) at NASA's Goddard Space Flight Center, we have made the first cryogenic measurements of absolute refractive index for Ohara L-BBH2 glass to enable the design of a prism for the Coronagraphic High Angular Resolution Imaging Spectrograph (CHARIS) at the Subaru telescope. L-BBH2 is employed in CHARIS's prism design for improving the spectrograph's dispersion uniformity. Index measurements were made at temperatures from 110 to $305 \mathrm{~K}$ at wavelengths from 0.46 to $3.16 \mu \mathrm{m}$. We report absolute refractive index (n), dispersion $(\mathrm{dn} / \mathrm{d} \lambda)$, and thermo-optic coefficient (dn/dT) for this material along with estimated single measurement uncertainties as a function of wavelength and temperature. We provide temperature-dependent Sellmeier coefficients based on our data to allow accurate interpolation of index to other wavelengths and temperatures within applicable ranges. This paper also speaks of the challenges in measuring index for a material which is not available in sufficient thickness to fabricate a typical prism for measurement in CHARMS, the tailoring of the index prism design that allowed these index measurements to be made, and the remarkable results obtained from that prism for this practical infrared material.
\end{abstract}

Keywords: L-BBH2, CHARMS, cryogenic refractive index, temperature-dependent, infrared, dispersion, thermo-optic coefficient, CHARIS, Subaru telescope

\section{INTRODUCTION}

The Cryogenic High Accuracy Refraction Measuring System (CHARMS) was developed at the NASA Goddard Space Flight Center (GSFC) to measure absolute refractive index (in vacuum) down to temperatures as low as $15 \mathrm{~K}$ with unsurpassed accuracy using minimum deviation refractometry. ${ }^{1-3}$ Initially, CHARMS was developed in support of the optical design for James Webb Space Telescope's (JWST) Near Infrared Camera (NIRCam) ${ }^{4}$ with an eye towards cryogenic refractive index measurements for a wide variety of infrared materials to serve the entire scientific community, both space-based and ground-based. To that end, CHARMS has supported a wide variety of international programs including JWST, the Keck Multi-Object Spectrometer for Infra-Red Exploration (MOSFIRE) ${ }^{5}$, the ESO UT1 CRyogenic high-resolution InfraRed Echelle Spectrograph (CRIRES) ${ }^{6}$ and VLT K-band Multi-Object Spectrograph (KMOS) ${ }^{7}$, the GMT Near Infrared Multi-Object Spectrograph (NIRMOS) ${ }^{8}$, the Euclid Near Infrared Spectrometer and Photometer (NISP $^{9,10}$, and NASA's Transiting Exoplanet Survey Satellite (TESS) ${ }^{11}$ and Wide Field Infrared Survey Telescope (WFIRST). ${ }^{12}$ CHARMS has now been used for measurements at wavelengths as low as $0.35 \mu \mathrm{m}$ in the near UV and to temperatures as high as $335 \mathrm{~K}$ (62 C). Figure 1 is a photograph of CHARMS at GSFC.

For the present study, we used CHARMS to measure absolute refractive indices covering wavelength and temperature ranges from 0.46 to $3.16 \mu \mathrm{m}$ and from 110 to $305 \mathrm{~K}$, respectively, for L-BBH2 - a relatively new, infrared glass from Ohara (a replacement for the now-obsolete L-BBH1) to enable the optimized optical design of dispersing prism elements for the CHARIS integral field spectrograph instrument for the Subaru telescope. ${ }^{13,14}$ The chief advantage of the high index L-BBH2 glass used in combination with low index barium fluoride in the instrument's prism design is that its dispersion allows for a relatively constant resolving power across the J, $\mathrm{H}$, and $\mathrm{K}$ infrared bands from $1.15-2.38 \mu \mathrm{m}$

* author contact: doug@levitonmetrology.com, 240-478-7877 
over both low and high resolution modes in CHARIS compared to the use of the best alternative material, S-FTM16. ${ }^{15,16}$ From a scientific perspective, the spectral resolving power achieved is quite beneficial in that it balances higher spectral resolution in the $\mathrm{J}$ band where it is needed and enhanced signal in the $\mathrm{K}$ band that would otherwise be undesirably overdispersed - an elegant balance of design goals.

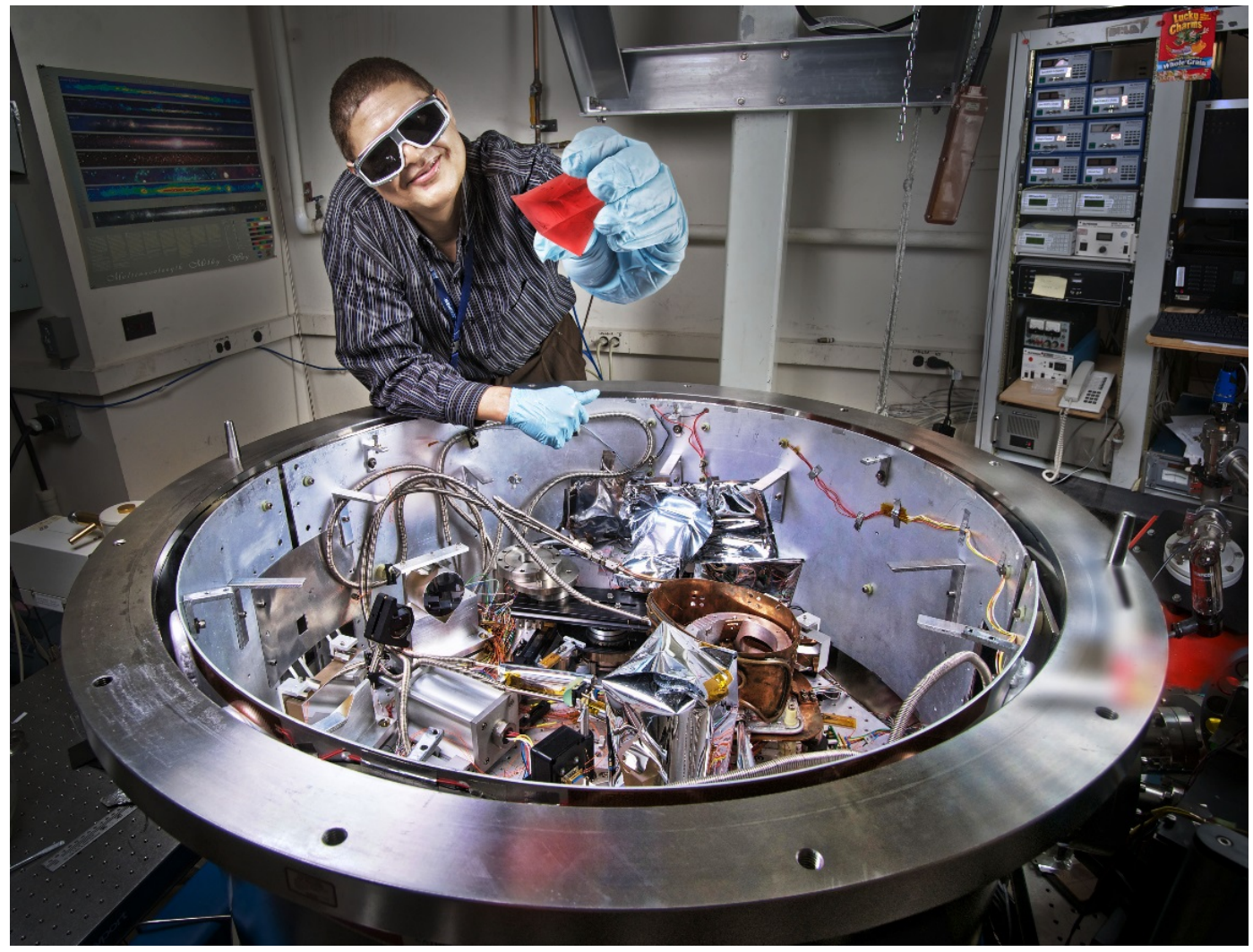

Figure 1 - CHARMS high accuracy, cryogenic refractometer at NASA GSFC (photo by Bert Pasquale and Scott Smith/GSFC)

\section{PRISM DESIGN CHALLENGES}

L-BBH2 material is not available from the manufacturer in sufficient thickness that the prism in CHARIS could be designed and made from a single piece of material. It is available only in sheet form nominally $10 \mathrm{~mm}$ in thickness. As a result, the L-BBH2 portion of the high resolution dispersing component in CHARIS is actually a pair of L-BBH2 prisms behaving as one optically.

Prisms measured in CHARMS are ordinarily designed to produce beam deviations either from $45^{\circ}$ to $64^{\circ}$ or from $20^{\circ}$ to $45^{\circ}$, depending on which of two CHARMS layouts is in use. Though switching from one layout to the other is not a trivial affair, the two layouts are due to CHARMS's being an absolute, cryogenic, minimum-deviation refractometer built to operate entirely inside a vacuum chamber and allow a wide of range of prism apex angles and refractive indices to be accommodated, generally using deviation angles which provide best index sensitivity. CHARMS's collimated beam from the monochromator's exit slit has a nominal width of $19 \mathrm{~mm}$ as it encounters a prism's input refracting face and has an image space $\mathrm{f} / \#$ of $\sim 55$ in the dispersion direction. The resulting slit image width gives acceptable precision for measuring deviation angle, and IR image irradiance provides acceptable signal over background.

A prism's top face must have sufficient area that a pair of temperature sensors can be attached, and its height is required to be $28 \mathrm{~mm}$. The prism is subjected to significant clamping force between a copper prism platform and a copper bus bar across the top of the prism, so avoiding a sliver-like prism minimizes stress at the apex of the optic, preserving good surface flatness and limiting risk of fracture at cryogenic temperatures. 
Given the lowest anticipated index that would have to be measured for L-BBH2 of $\sim 2.0$, an apex angle for the prism of at least $39^{\circ}$ would be required to deviate beams into CHARMS's higher accessible range of angular deviations in use at the time these measurements of L-BBH2 were conceived. With the prism material thickness limitation of only $10 \mathrm{~mm}$, however, the aperture needed to limit the beam to the optically flat parts of the input surface for a prism with that apex angle would be rather small at only $8 \mathrm{~mm}$ wide. Meanwhile, before L-BBH2 index measurements actually commenced, another index measurement campaign required CHARMS to be reconfigured for the smaller range of accessible deviation angles. This was fortunate as it allowed us to design a prism with an apex angle of only $28^{\circ}$, affording a significantly wider input beam width of $14 \mathrm{~mm}$.

Figure 3 illustrates the negative consequences of having too small a beam width in CHARMS. In this figure, simulated relative signal at a wavelength of $2 \mu \mathrm{m}$ is plotted against relative deviated beam angle for $19 \mathrm{~mm}, 14 \mathrm{~mm}$, and $8 \mathrm{~mm}$ beam widths (Figure 2, left, right, and middle, respectively). For the signal simulation, first, a slit image profile is approximated as a tophat function representing the $350 \mu \mathrm{m}$ geometric image width of the monochromator exit slit with a half-Gaussian profile of half-width equal to half the diffraction spot size that pertains to each beam width (and its associated $\mathrm{f} / \#$ ) appended to each side of that tophat.

For a wavelength of $2 \mu \mathrm{m}$, a $19 \mathrm{~mm}$ beam has a diffraction image width of $\sim 200 \mu \mathrm{m}$, a $14 \mathrm{~mm}$ beam has a diffraction image width of $\sim 300 \mu \mathrm{m}$, and an $8 \mathrm{~mm}$ beam has a diffraction image width of $\sim 500 \mu \mathrm{m}$. A detector signal scan profile is then created by convolving this slit image profile with a $300 \mu \mathrm{m}$ detector slit. (The scan profiles for $14 \mathrm{~mm}$ and $19 \mathrm{~mm}$ beams in Figure 3 are a good match to actual measured scan profiles for those aperture widths.) After the scan profile is simulated, the amplitude of the profile is adjusted to account for the attenuation of available energy in the collimated beam associated with the each aperture area. With an $8 \mathrm{~mm}$ wide aperture, IR signal levels would be expected to be only $42 \%$ of what they would be for a $19 \mathrm{~mm}$ wide aperture. With a $14 \mathrm{~mm}$ wide beam, relative signal levels would be at the $74 \%$ level.

For the $8 \mathrm{~mm}$ beam, the slit scan function widens dramatically at the same time that irradiance at the measurement slit in front of the IR detector drops. Measuring beam deviation angle via the centroid of the scan profile becomes less deterministic, and noise on the slit scan signal becomes more prevalent. The $14 \mathrm{~mm}$ beam used in our index measurements of L-BBH2 yielded a good compromise between scan profile width and signal level.
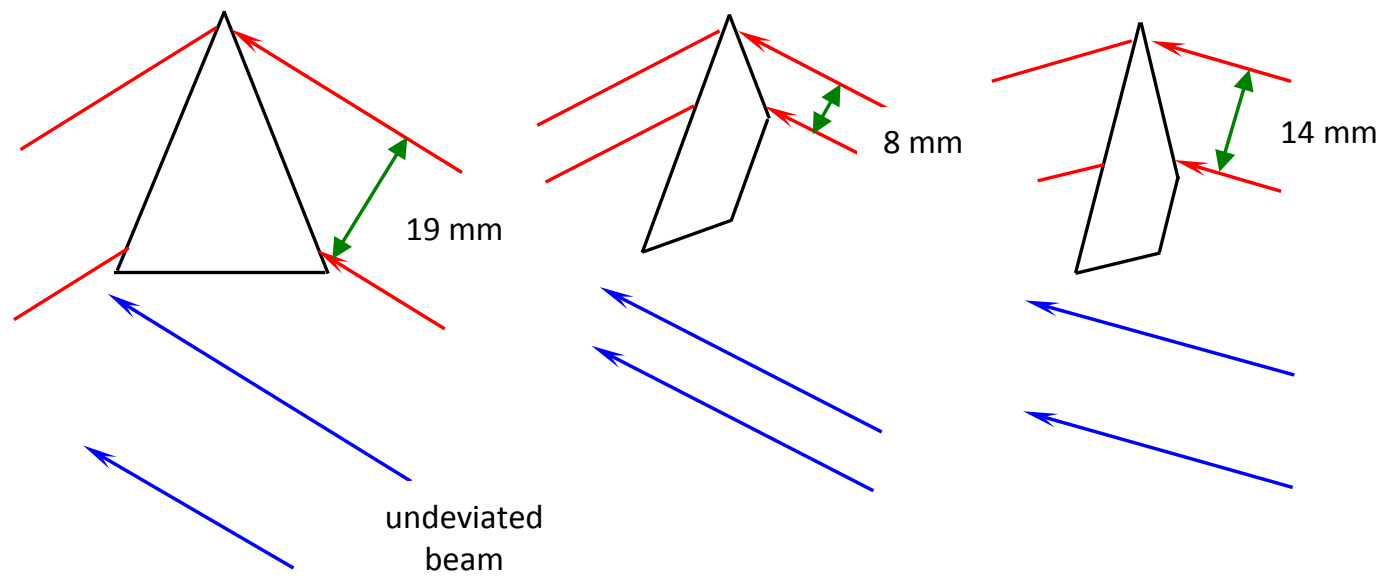

Figure 2 - beam geometries for different prism designs; red lines are beams refracted through prism; blue lines are the undeviated beam which passes behind the prism in CHARMS; green lines indicate beam width; left) ideal design for CHARMS prism made from L-BBH2 has a $44^{\circ}$ apex angle and $38 \mathrm{~mm}$ long refracting faces, enjoys beam apertures $19 \mathrm{~mm}$ in diameter, and deviates the input beam by $64^{\circ}$ at maximum index of 2.2; middle) prism design limited by $10 \mathrm{~mm}$ nominal available material thickness for higher range of accessible beam deviation angles has a $40^{\circ}$ apex angle and $16 \mathrm{~mm} / 35 \mathrm{~mm}$ long input/output refracting faces, but uses beam apertures only $8 \mathrm{~mm}$ wide, and deviates the input beam by $54^{\circ}$ at maximum index of 2.2 and by $46^{\circ}$ at index of 2.0 - just inside the higher accessible range of deviation angles; right) more optimal prism design, again limited by 10 mm nominal available material thickness for lower range of accessible beam deviation angles has a $28^{\circ}$ apex angle and $21 \mathrm{~mm} / 38 \mathrm{~mm}$ long input/output refracting faces, uses beam apertures that are $14 \mathrm{~mm}$ wide, and deviates the input beam by $36^{\circ}$ at maximum index of 2.2 and by $30^{\circ}$ at index of 2.0; this is the prism used for index measurements in this study 


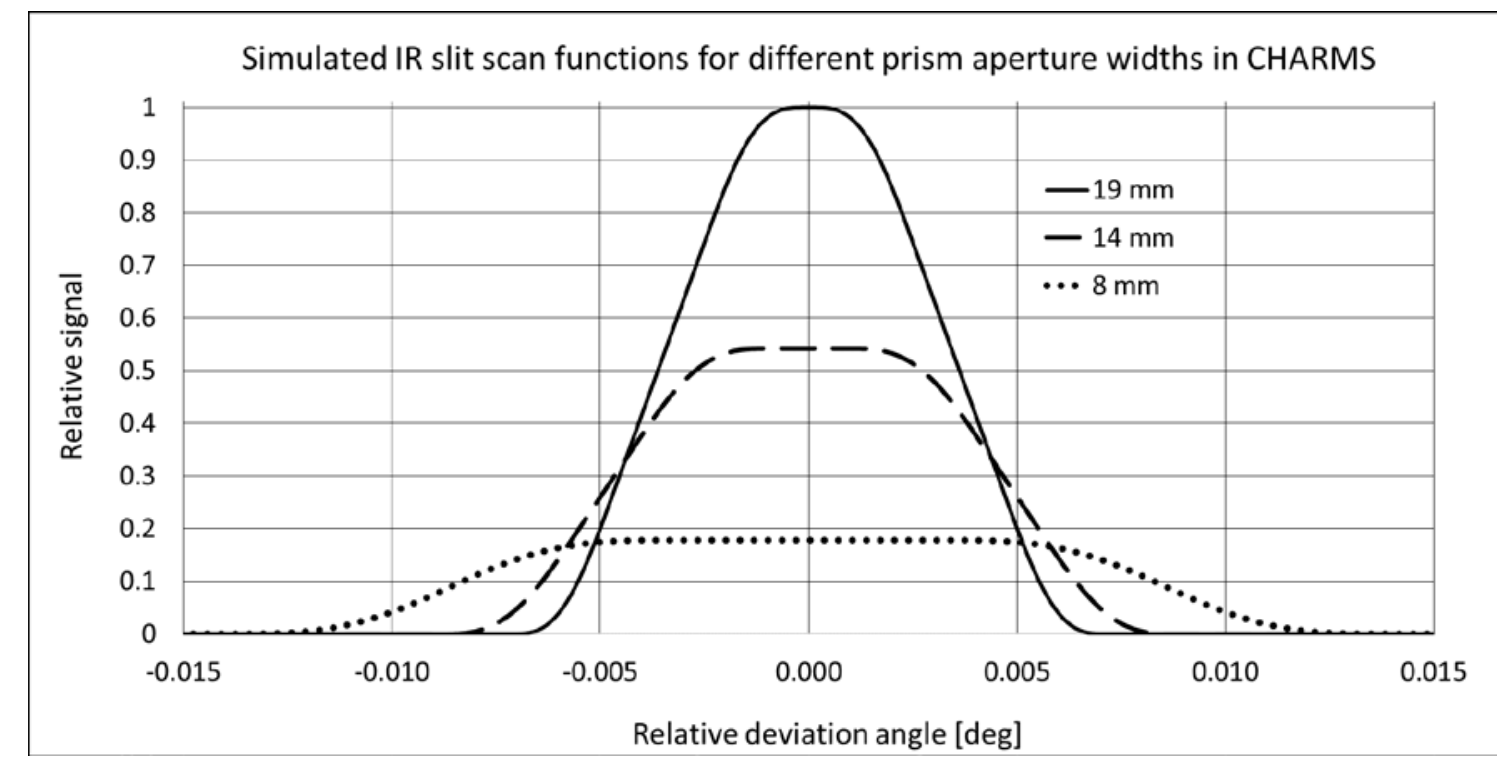

Figure 3 - with decreased beam width, the CHARMS slit scan function for $2 \mu$ m wavelength widens dramatically at the same time that irradiance at the IR detector slit drops; the combined effect of these things could reduce certainty in measuring beam deviation angle as the centroid of the scan profile becomes less deterministic, and noise increases

\section{PRESENTATION OF MEASURED INDEX DATA}

Detailed descriptions of our data acquisition and reduction processes are documented elsewhere ${ }^{17}$, as is our calibration strategy ${ }^{1}$. We fit our raw, measured index data to a temperature dependent Sellmeier model of the form:

$$
\mathrm{n}^{2}(\lambda, \mathrm{T})-1=\sum_{\mathrm{i}=1}^{\mathrm{m}} \frac{\mathrm{S}_{\mathrm{i}}(\mathrm{T}) \cdot \lambda^{2}}{\lambda^{2}-\lambda_{\mathrm{i}}^{2}(\mathrm{~T})},
$$

where $S_{i}$ are theoretical strengths of resonance features in the material at wavelengths $\lambda_{i}$. When dealing with a wavelength interval between wavelengths of physical resonances in a material, the summation may be approximated by typically only three terms $(\mathrm{m}=3) .{ }^{18}$ With this approximation, resonance strengths $S_{\mathrm{i}}$ and wavelengths $\lambda_{\mathrm{i}}$ have no physical significance but are simply parameters used to generate adequately accurate fits to measured data. If these parameters are assumed to be functions of temperature, $T$, one can generate a temperature-dependent Sellmeier model for $n(\lambda, T)$.

Historically, this modeling approach has enjoyed significant success for a variety of materials despite a rather serious sparseness of available index data to cover a wide range of temperatures and wavelengths upon which to base a model. Sadly, some designers have experienced disappointing performance in as-built lenses by extrapolating outside the ranges of applicability of published Sellmeier models. One solution to lacking measured index data has been to appeal to room temperature refractive index data at several wavelengths to anchor a model and then to extrapolate index values for other temperatures using accurate measurements of the thermo-optic coefficient at those temperatures which are much easier to make than accurate measurements of the index itself at exotic temperatures. Such extrapolation can be potentially dangerous, depending on the sample material in question and the required accuracy of index knowledge.

Meanwhile, with CHARMS, we directly measure index itself, densely sampling over a wide range of wavelengths and temperatures to produce a model with residuals on the order of (usually significantly less than) the uncertainties in our raw index measurements. For the model for L-BBH2, we found that 3rd order temperature dependencies in all three terms in each of $S_{i}$ and $\lambda_{i}$ are adequate. The Sellmeier equation consequently becomes:

$$
\mathrm{n}^{2}(\lambda, T)-1=\sum_{\mathrm{i}=1}^{3} \frac{\mathrm{S}_{\mathrm{i}}(\mathrm{T}) \cdot \lambda^{2}}{\lambda^{2}-\lambda_{\mathrm{i}}^{2}(\mathrm{~T})} \quad \text { where, }
$$




$$
\begin{aligned}
& S_{i}(T)=\sum_{j=0}^{3} S_{i j} \cdot T^{j} \\
& \lambda_{i}(T)=\sum_{j=0}^{3} \lambda_{i j} \cdot T^{j}
\end{aligned}
$$

The Sellmeier model is our best statistical representation of the raw, measured index data over the complete measured ranges of wavelength and temperature and allows accurate computation of the derivatives of index with respect to those two parameters. All tabulated values for refractive index and its derivatives have been calculated using the Sellmeier coefficients found later in Section 5. Typically, measured index values agree with the fits to less than our measurement uncertainties found in Section 4. Three things to remember when applying these Sellmeier fits and coefficients: 1) do not attempt to apply the fit outside the stated range of applicability; 2) test the fit to assure you can reproduce any value in Table 3; and 3) make sure to use all significant figures listed for each coefficient.

\section{MEASUREMENT UNCERTAINTIES}

CHARMS was designed specifically to minimize all sources of systematic errors in refractive index measurement and to provide measurements of highest achievable precision at both ambient and cryogenic temperatures. A non-exhaustive discussion which touches on the four most significant possible contributors to index errors has been published earlier. ${ }^{15}$ Since refractive index is a function of wavelength and temperature, likewise so is index measurement uncertainty.

Table 1 summarizes estimated uncertainty for single index measurements for the thin L-BBH2 prism in this study having $28^{\circ}$ apex angle using a $14 \mathrm{~mm}$ beam. Figure 4 is a surface plot of uncertainty values in the table. Note how uncertainty rises precipitously at shorter wavelengths where dispersion is higher and at low temperatures where gradients in temperature in the sample are hard to eliminate, especially for glasses which tend to have low thermal conductivity, and $\mathrm{dn} / \mathrm{dT}$ is high. CHARIS requires index knowledge of $1 \mathrm{E}-4$ from 1.15 to $2.38 \mu \mathrm{m}$.

Table 1 - Uncertainty of absolute refractive index of L-BBH2 in CHARMS for selected wavelengths \& temperatures

\begin{tabular}{|c|c|c|c|c|c|}
\hline wavelength $[\mu \mathrm{m}]$ & $120 \mathrm{~K}$ & $150 \mathrm{~K}$ & $200 \mathrm{~K}$ & $250 \mathrm{~K}$ & $300 \mathrm{~K}$ \\
\hline 0.5 & $1.1 \mathrm{E}-04$ & $1.0 \mathrm{E}-04$ & $1.0 \mathrm{E}-04$ & $8.3 \mathrm{E}-05$ & $5.8 \mathrm{E}-05$ \\
\hline 0.63 & $5.7 \mathrm{E}-05$ & $5.3 \mathrm{E}-05$ & $5.3 \mathrm{E}-05$ & $4.2 \mathrm{E}-05$ & $2.5 \mathrm{E}-05$ \\
\hline 1 & $2.8 \mathrm{E}-05$ & $2.8 \mathrm{E}-05$ & $3.0 \mathrm{E}-05$ & $2.3 \mathrm{E}-05$ & $1.1 \mathrm{E}-05$ \\
\hline 2 & $2.9 \mathrm{E}-05$ & $3.1 \mathrm{E}-05$ & $3.3 \mathrm{E}-05$ & $2.8 \mathrm{E}-05$ & $1.8 \mathrm{E}-05$ \\
\hline 2.8 & $3.0 \mathrm{E}-05$ & $3.1 \mathrm{E}-05$ & $3.3 \mathrm{E}-05$ & $1.7 \mathrm{E}-05$ & $1.8 \mathrm{E}-05$ \\
\hline
\end{tabular}

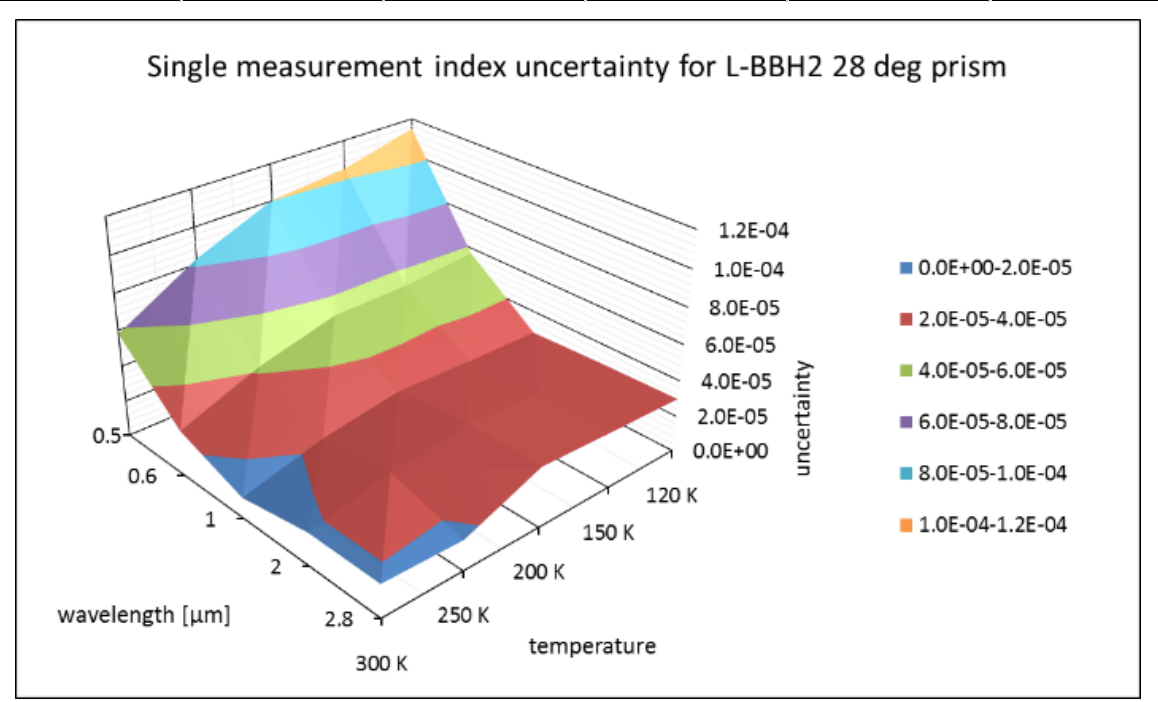

Figure 4 - single measurement uncertainty in refractive index of L-BBH2 prism with $28^{\circ}$ apex; note how uncertainty rises precipitously at low temperatures and short wavelengths 


\section{MEASUREMENT RESULTS}

The coefficients for a temperature-dependent Sellmeier fit of measured index to wavelength and temperature (to be used as prescribed in Section 3) based on 2,813 individual index measurements are listed in Table 2. The sensitivity of index accuracy when using the fit to the number of significant digits for each coefficient is unknown, so nine significant digits are reported for all coefficients (very likely more than needed). The average, absolute residual of the measured data from the fit is $9.2 \mathrm{E}-6$ considering all measured indices and 7.9E-6 considering only measured indices within the CHARIS spectral bands. Tables 3, 4, and 5 contain absolute refractive indices of L-BBH2 computed from the Sellmeier fit along with its wavelength and temperature derivatives - spectral dispersion, $\mathrm{dn} / \mathrm{d} \lambda$, and thermo-optic coefficient, $\mathrm{dn} / \mathrm{dT}$, respectively (CHARIS's IR bands are shaded in colors showing band center and end wavelengths in Table 3). Figures 5, 6, and 7 are graphs of spectral index, dispersion, and thermo-optic coefficient, respectively.

Table 2 - Coefficients for temperature-dependent Sellmeier fit of absolute refractive index of L-BBH2

\begin{tabular}{|c|c|c|c|c|c|c|}
\hline \multicolumn{7}{|c|}{ Coefficients for temperature-dependent Sellmeier equation for L-BBH2 } \\
$\mathbf{1 1 0} \mathbf{K} \leq \mathbf{T} \leq \mathbf{3 0 5} \mathbf{~ K} \mathbf{0 . 4 6} \boldsymbol{\mu m} \leq \boldsymbol{\lambda} \leq \mathbf{3 . 1 6} \boldsymbol{\mu m}$ \\
\hline \\
\hline
\end{tabular}

Table 3 - Computed absolute refractive index of L-BBH2 for selected wavelengths and temperatures

\begin{tabular}{|c|c|c|c|c|c|}
\hline wavelength $[\mu \mathrm{m}]$ & $110 \mathrm{~K}$ & $150 \mathrm{~K}$ & $200 \mathrm{~K}$ & $250 \mathrm{~K}$ & $300 \mathrm{~K}$ \\
\hline 0.416 & 2.20330 & 2.20427 & 2.20599 & 2.20812 & 2.21055 \\
\hline 0.50 & 2.13531 & 2.13584 & 2.13683 & 2.13810 & 2.13962 \\
\hline 0.633 & 2.08880 & 2.08913 & 2.08977 & 2.09062 & 2.08791 \\
\hline 1.00 & 2.04783 & 2.04801 & 2.04842 & 2.04900 & 2.04973 \\
\hline 1.15 & 2.04088 & 2.04104 & 2.04141 & 2.04196 & 2.04265 \\
\hline 1.23 & 2.03797 & 2.03812 & 2.03848 & 2.03902 & 2.03970 \\
\hline 1.31 & 2.03543 & 2.03557 & 2.03593 & 2.03645 & 2.03712 \\
\hline 1.50 & 2.03034 & 2.03047 & 2.03081 & 2.03132 & 2.03197 \\
\hline 1.63 & 2.02733 & 2.02746 & 2.02779 & 2.02829 & 2.02893 \\
\hline 1.76 & 2.02452 & 2.02464 & 2.02497 & 2.02547 & 2.02610 \\
\hline 2.00 & 2.01957 & 2.01969 & 2.02002 & 2.02051 & 2.02113 \\
\hline 2.19 & 2.01567 & 2.01580 & 2.01612 & 2.01661 & 2.01723 \\
\hline 2.38 & 2.01167 & 2.01179 & 2.01212 & 2.01260 & 2.01322 \\
\hline 2.80 & 2.00204 & 2.00218 & 2.00251 & 2.00299 & 2.00361 \\
\hline 3.20 & 1.99144 & 1.99161 & 1.99196 & 1.99245 & 1.99307 \\
\hline
\end{tabular}

Table 4 - Dispersion in L-BBH2 for selected wavelengths and temperatures (units $1 / \mu \mathrm{m}$ )

\begin{tabular}{|c|c|c|c|c|c|}
\hline wavelength $[\mu \mathrm{m}]$ & $110 \mathrm{~K}$ & $150 \mathrm{~K}$ & $200 \mathrm{~K}$ & $250 \mathrm{~K}$ & $300 \mathrm{~K}$ \\
\hline 0.45 & -0.8094 & -0.8146 & -0.8233 & -0.8335 & -0.8444 \\
\hline 0.50 & -0.5607 & -0.5637 & -0.5688 & -0.5747 & -0.5814 \\
\hline 0.55 & -0.3834 & -0.3852 & -0.3881 & -0.3916 & -0.3956 \\
\hline 0.60 & -0.2775 & -0.2786 & -0.2806 & -0.2829 & -0.2855 \\
\hline 0.70 & -0.1628 & -0.1634 & -0.1644 & -0.1656 & -0.1669 \\
\hline 0.80 & -0.1061 & -0.1065 & -0.1070 & -0.1077 & -0.1085 \\
\hline 0.90 & -0.0745 & -0.0748 & -0.0752 & -0.0756 & -0.0761 \\
\hline 1.00 & -0.0556 & -0.0558 & -0.0560 & -0.0563 & -0.0567 \\
\hline 1.25 & -0.0328 & -0.0329 & -0.0330 & -0.0332 & -0.0333 \\
\hline 1.50 & -0.0244 & -0.0245 & -0.0245 & -0.0246 & -0.0247 \\
\hline 2.00 & -0.0205 & -0.0205 & -0.0205 & -0.0205 & -0.0205 \\
\hline 2.20 & -0.0208 & -0.0208 & -0.0208 & -0.0208 & -0.0209 \\
\hline 2.40 & -0.0217 & -0.0217 & -0.0217 & -0.0217 & -0.0217 \\
\hline 3.00 & -0.0265 & -0.0264 & -0.0264 & -0.0264 & -0.0264 \\
\hline
\end{tabular}


Table 5 - Thermo-optic coefficient (dn/dT) of L-BBH2 for selected wavelengths and temperatures (units 1/K)

\begin{tabular}{|c|c|c|c|c|c|}
\hline wavelength $[\mu \mathrm{m}]$ & $120 \mathrm{~K}$ & $150 \mathrm{~K}$ & $200 \mathrm{~K}$ & $250 \mathrm{~K}$ & $290 \mathrm{~K}$ \\
\hline 0.42 & $2.16 \mathrm{E}-05$ & $2.93 \mathrm{E}-05$ & $3.88 \mathrm{E}-05$ & $4.59 \mathrm{E}-05$ & $5.02 \mathrm{E}-05$ \\
\hline 0.45 & $1.63 \mathrm{E}-05$ & $2.22 \mathrm{E}-05$ & $2.98 \mathrm{E}-05$ & $3.61 \mathrm{E}-05$ & $4.04 \mathrm{E}-05$ \\
\hline 0.50 & $1.19 \mathrm{E}-05$ & $1.65 \mathrm{E}-05$ & $2.27 \mathrm{E}-05$ & $2.80 \mathrm{E}-05$ & $3.19 \mathrm{E}-05$ \\
\hline 0.55 & $9.34 \mathrm{E}-06$ & $1.33 \mathrm{E}-05$ & $1.87 \mathrm{E}-05$ & $2.34 \mathrm{E}-05$ & $2.68 \mathrm{E}-05$ \\
\hline 0.60 & $7.72 \mathrm{E}-06$ & $1.13 \mathrm{E}-05$ & $1.62 \mathrm{E}-05$ & $2.05 \mathrm{E}-05$ & $2.36 \mathrm{E}-05$ \\
\hline 0.65 & $6.60 \mathrm{E}-06$ & $9.91 \mathrm{E}-06$ & $1.45 \mathrm{E}-05$ & $1.85 \mathrm{E}-05$ & $2.14 \mathrm{E}-05$ \\
\hline 0.70 & $5.79 \mathrm{E}-06$ & $8.91 \mathrm{E}-06$ & $1.33 \mathrm{E}-05$ & $1.70 \mathrm{E}-05$ & $1.98 \mathrm{E}-05$ \\
\hline 0.80 & $4.71 \mathrm{E}-06$ & $7.57 \mathrm{E}-06$ & $1.16 \mathrm{E}-05$ & $1.52 \mathrm{E}-05$ & $1.77 \mathrm{E}-05$ \\
\hline 0.90 & $4.02 \mathrm{E}-06$ & $6.72 \mathrm{E}-06$ & $1.06 \mathrm{E}-05$ & $1.40 \mathrm{E}-05$ & $1.64 \mathrm{E}-05$ \\
\hline 1.00 & $3.55 \mathrm{E}-06$ & $6.16 \mathrm{E}-06$ & $9.93 \mathrm{E}-06$ & $1.32 \mathrm{E}-05$ & $1.56 \mathrm{E}-05$ \\
\hline 1.20 & $2.97 \mathrm{E}-06$ & $5.46 \mathrm{E}-06$ & $9.11 \mathrm{E}-06$ & $1.23 \mathrm{E}-05$ & $1.46 \mathrm{E}-05$ \\
\hline 1.40 & $2.62 \mathrm{E}-06$ & $5.08 \mathrm{E}-06$ & $8.66 \mathrm{E}-06$ & $1.18 \mathrm{E}-05$ & $1.40 \mathrm{E}-05$ \\
\hline 1.80 & $2.28 \mathrm{E}-06$ & $4.73 \mathrm{E}-06$ & $8.26 \mathrm{E}-06$ & $1.13 \mathrm{E}-05$ & $1.34 \mathrm{E}-05$ \\
\hline 2.40 & $2.32 \mathrm{E}-06$ & $4.72 \mathrm{E}-06$ & $8.15 \mathrm{E}-06$ & $1.11 \mathrm{E}-05$ & $1.32 \mathrm{E}-05$ \\
\hline 3.20 & $3.68 \mathrm{E}-06$ & $5.53 \mathrm{E}-06$ & $8.32 \mathrm{E}-06$ & $1.11 \mathrm{E}-05$ & $1.34 \mathrm{E}-05$ \\
\hline
\end{tabular}

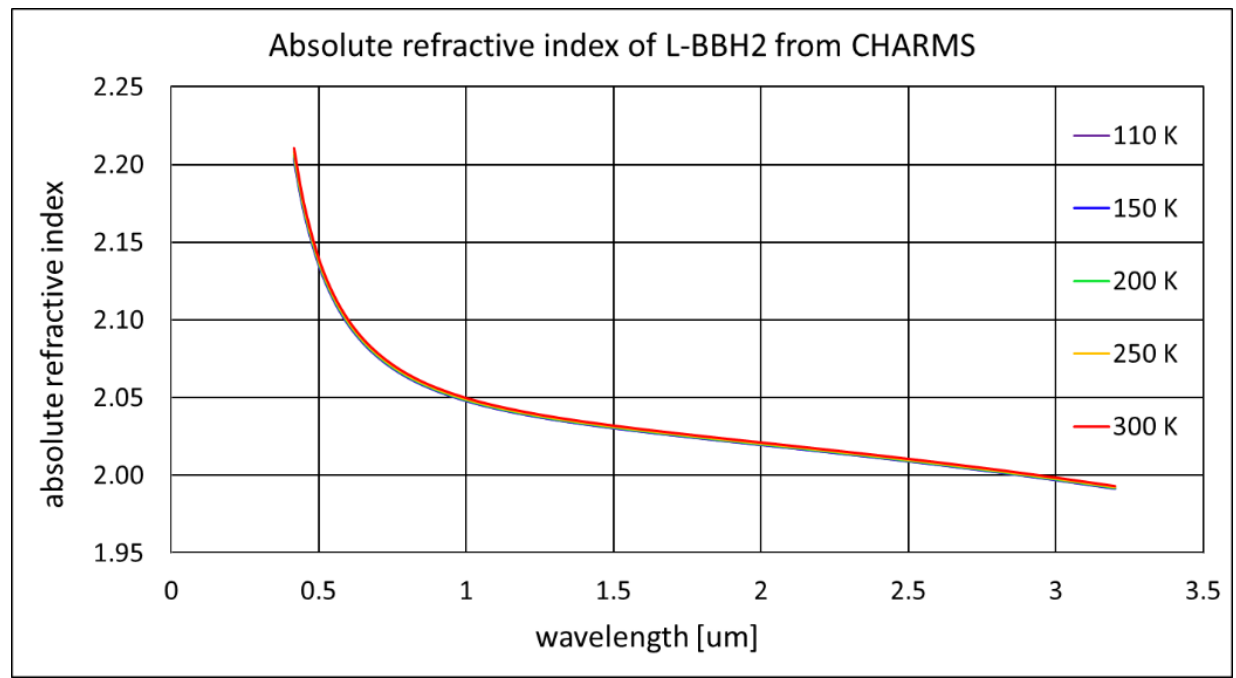

Figure 5 -Absolute spectral refractive index n of L-BBH2 for selected temperatures

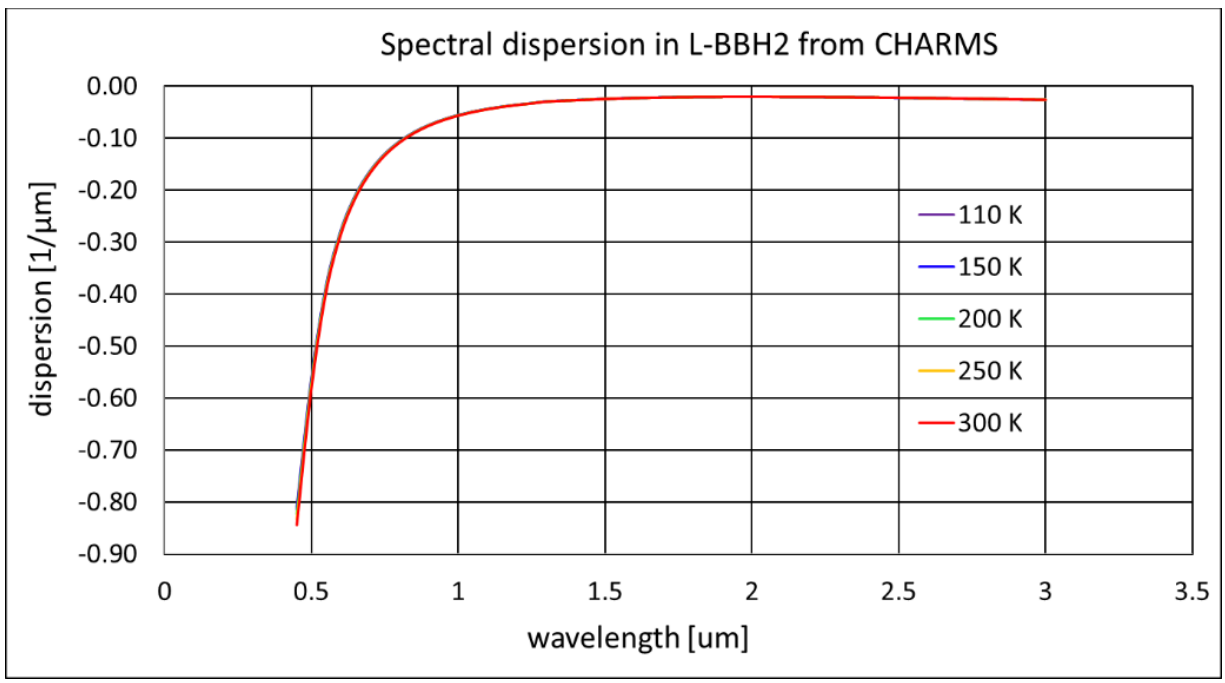

Figure 6 - Spectral dispersion $(\mathrm{dn} / \mathrm{d} \lambda)$ of L-BBH2 for selected temperatures 


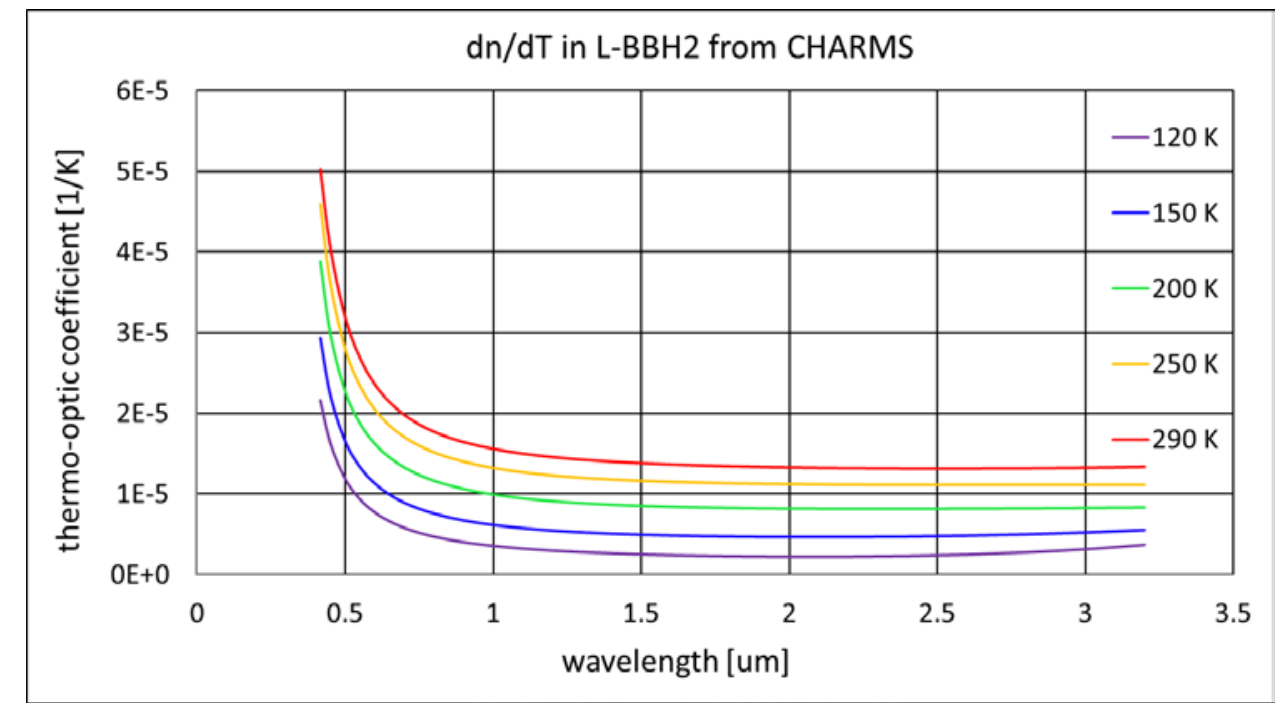

Figure 7 - Spectral thermo-optic coefficient (dn/dT) of L-BBH2 for selected temperatures

\section{COMPARISON WITH CATALOG VALUES}

Often, we find little or no refractive index data at cryogenic temperatures with which to compare our CHARMS measurements - and L-BBH2 is no exception - so, we can at least compare our measurements with the manufacturer's $\left(\right.$ Ohara Corporation ${ }^{19}$ ) published room temperature dispersion relation. Ohara does not currently specify temperature for this dispersion relation in the data sheet or on its website, so we will assume that room temperature is $25 \mathrm{C}$ in air as it has been in the past for other Ohara data. We first adjust Ohara's catalog indices to vacuum by multiplying by the spectral index of refraction of air. Indices from CHARMS are then computed at $298 \mathrm{~K}$ (25 C) for comparison.

Figure 8 shows index difference as CHARMS minus Ohara. On balance, it appears that the melt of L-BBH2 tested is well represented by the catalog dispersion law. The difference between CHARMS and Ohara is a smooth curve that somewhat resembles the spectral index curve itself suggesting some systematic difference between Ohara's index measurements and CHARMS of order +/-8E-5 peak- nearly double our uncertainty at $0.5 \mu \mathrm{m}$ and $\sim 4 \mathrm{X}$ our uncertainty at $2.5 \mu \mathrm{m}$. It is still true that when there is no knowledge of index behavior over a wide temperature range - especially to cryogenic temperatures - if for one's optical design and build, one needs to know a material's refractive index to an accuracy better than the known variability in the material, it is best practice to purchase enough material for the project from a given melt(s), make a prism(s) from the lot of material, and measure refractive indices in the same environment that the optics will see in use. Numerous optical designs have had excellent success realizing expected performance without repeat integration and test cycles, the cost of which easily dwarfs the cost of such index measurements. Plan ahead and allow time for refractive index to be measured well in advance of the final design and fabrication of the optics.

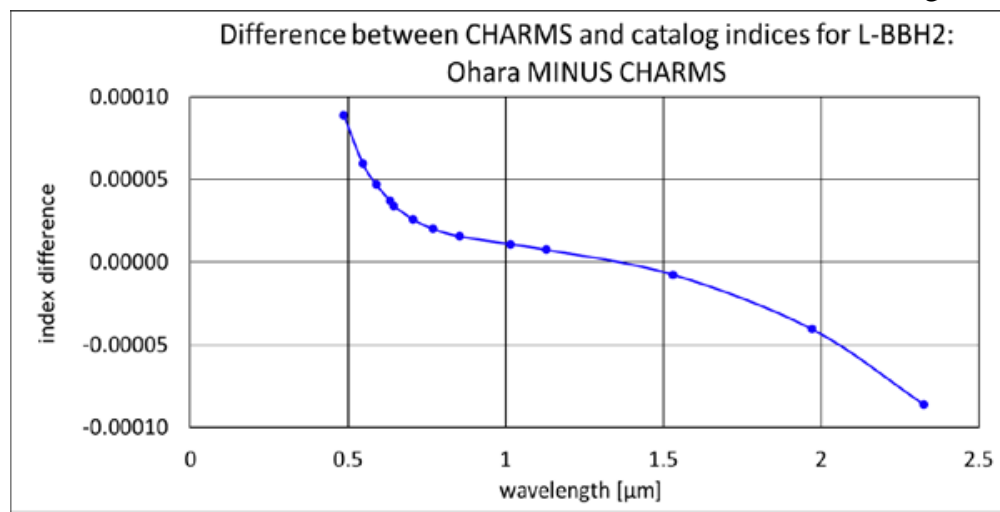

Figure 8 - Ohara L-BBH2 catalog indices for L-BBH2 minus CHARMS computed indices at 298 K (25 C) 


\section{CONSIDERATIONS FOR CHARIS}

Figure 9 illustrates the basic differences between the dispersion of L-BBH2 and the alternative material for THE CHARIS disperser prism, S-FTM16, in the J, H, and K infrared bands from $1.15-2.4 \mu \mathrm{m}$ at $110 \mathrm{~K}$. It is clear how LBBH2 is advantageous to CHARIS in that it has $2.4 \mathrm{X}$ as much dispersion as S-FTM16 at the shorter wavelengths where it is needed and only $1.25 \mathrm{X}$ as much dispersion as S-FTM16 at the longer wavelengths.

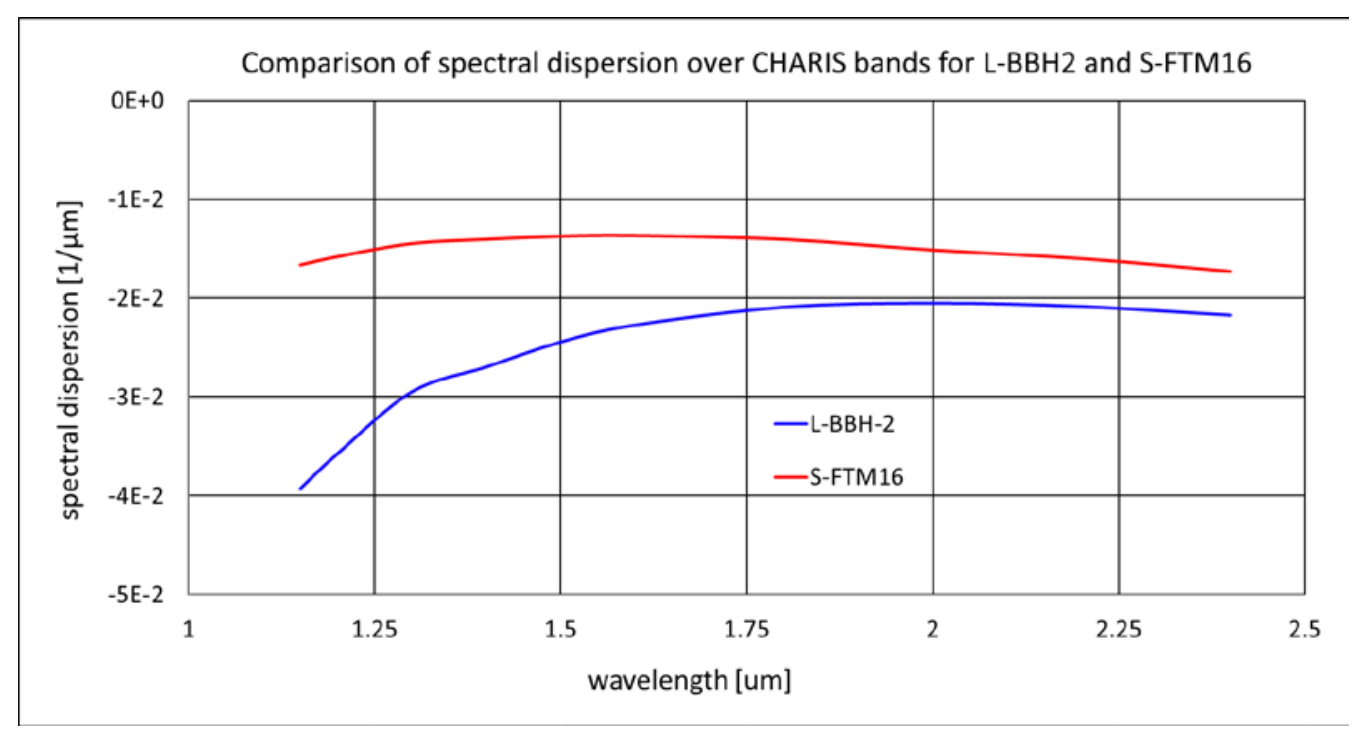

Figure 9 - comparison of dispersion in L-BBH2 and S-FTM16 glasses

CHARIS is designed to operate at temperatures as low as $70 \mathrm{~K}$, yet our ability to measure with LHe to get the sample that cold was unavailable at the time these CHARMS index measurements were made. Thus, we were only able to get the sample down to $110 \mathrm{~K}$ using LN2 - $40 \mathrm{~K}$ above the low end of the CHARIS operating temperature range. While we are loathe to extrapolate measured index beyond the measured range of temperature, in the case of L-BBH2, we are able to bound the amount of index error in doing so because, fortunately, L-BBH2 has particularly low and diminishing thermo-optic coefficient $(\mathrm{dn} / \mathrm{dT})$ in the IR at temperatures below $120 \mathrm{~K}$.

Note that to perform the extrapolation, we do not extrapolate using the Sellmeier fit which might have pathological behavior outside of its range of applicability. Instead, we "go old school" and actually use a French curve laid over index plots to predict indices to colder temperatures. In doing so, to set a lower bound on index at temperatures below $110 \mathrm{~K}$, we do as many optical designers do and offset the index at the lowest temperature measured by the product of the thermo-optic coefficient, $\mathrm{dn} / \mathrm{dT}$, at that temperature and a negative temperature interval. This is a valid thing to do since $\mathrm{dn} / \mathrm{dT}$ below $110 \mathrm{~K}$ is monotonically approaching zero, and extrapolating using a French curve is capable of producing a more accurate index by accounting for this behavior. Meanwhile, assuming that the measured index at $110 \mathrm{~K}$ also applies to lower temperatures sets the upper bound for index below $110 \mathrm{~K}$. These two methods produce a band of index values whose width ideally does not exceed our index knowledge requirement of $+/-1 \mathrm{E}-4$.

Figure 10 shows the width of the index band between upper and lower bounds from extrapolation methods as a function of wavelength and temperatures colder than $110 \mathrm{~K}$. It should be obvious that the shape of the band at each temperature matches the shape of $\mathrm{dn} / \mathrm{dT}$ since one of the two bounds is a constant, and in the short wavelength region where $\mathrm{dn} / \mathrm{dT}$

rises precipitously, the width of the band so grows rapidly. Over the wavelength interval from 1.15 to $2.38 \mu \mathrm{m}$ for the J, $\mathrm{H}$, and $\mathrm{K}$ bands of CHARIS, only the index band curve for $70 \mathrm{~K}$ has a width that exceeds $1 \mathrm{E}-4$. This implies that the French curve extrapolation which lies essentially in the middle of the band would be in error by no more than half the width of the band. Thus, that French curve extrapolation should provide adequate knowledge of index, even at $70 \mathrm{~K}$. The plotted points in Figure 10 are located at the J, H, and $\mathrm{K}$ band centers and are scaled on the right ordinate. These points illustrate positive margin on index knowledge for CHARIS even down to $70 \mathrm{~K}$. 


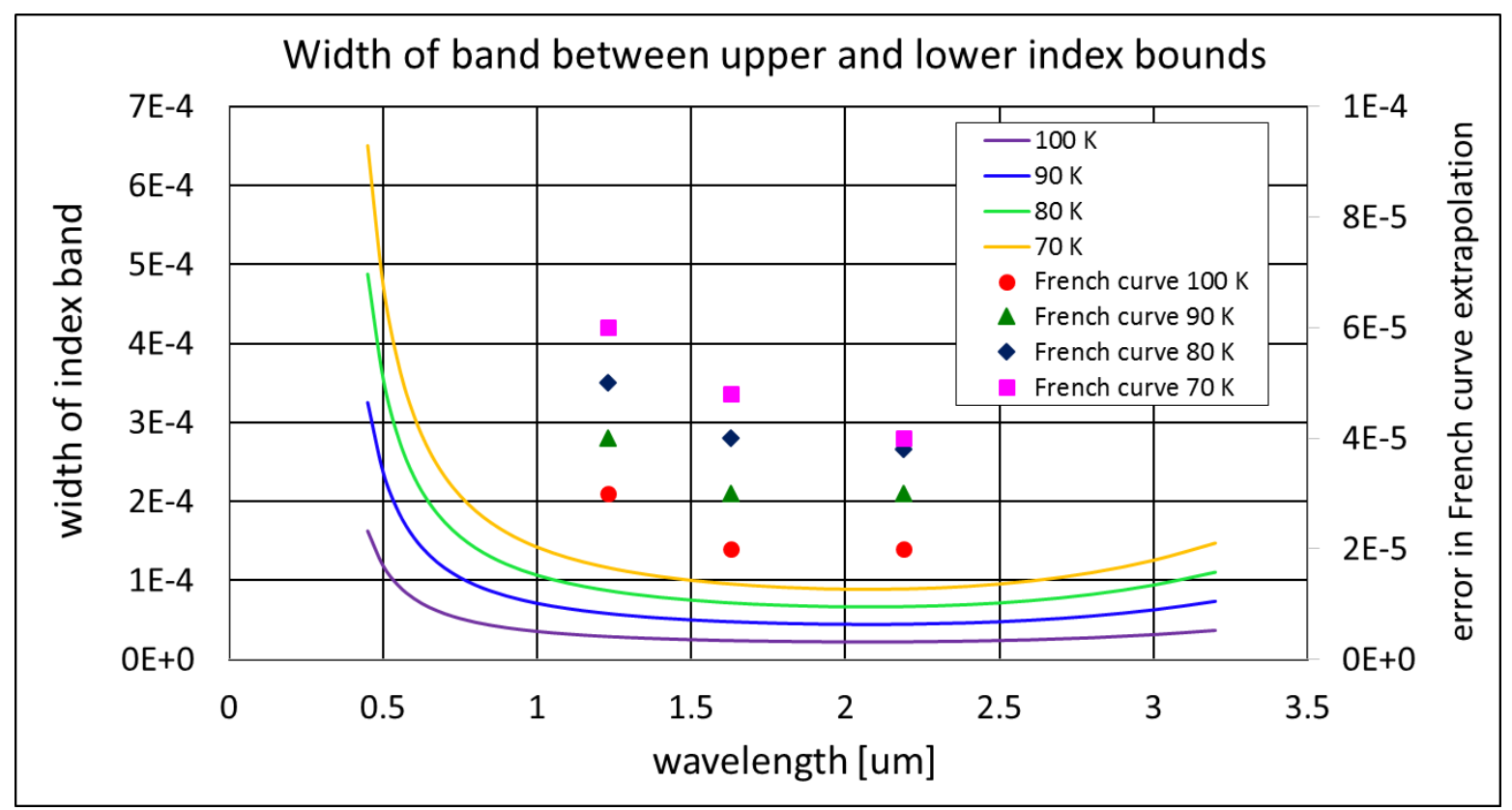

Figure 10 - width of band between upper and lower index bounds using worst case extrapolation methods; French curve extrapolation lies in the middle of the band and yields the most accurate index values at temperatures below $110 \mathrm{~K}$ where CHARIS is designed to operate

\section{CONCLUSION}

We have measured the absolute refractive indices of the Ohara glass L-BBH2 for the first time from the blue to $3.16 \mu \mathrm{m}$ in the infrared from room temperature down to $110 \mathrm{~K}$ to support the design of dispersing prism components for the CHARIS instrument at the Subaru telescope. We see that catalog data is clearly insufficient for knowing what index to expect at the $1 \mathrm{E}-4$ level of index for temperatures well-removed from room temperature.

We discussed the challenges of tailoring a prism for measurement in CHARMS when raw material is not available in a form thick enough to fabricate the ideal index prism. We compared our CHARMS measurements to the glass manufacturer's index data at room temperature and compared the spectral dispersion of L-BBH2 to that of the best alternative material for CHARIS dispersing prisms, S-FTM16.

Though we did not quite reach the operating temperature range of CHARIS in our measurements, fortuitously, the index of L-BBH2 stops changing at temperatures below those measured to the degree that adequate index accuracy in that operating temperature range can be obtained by extrapolation of index curves using a French curve.

\section{REFERENCES}

[1] Leviton, D.B., Frey, B.J., "Design of a cryogenic, high accuracy, absolute prism refractometer for infrared through far ultraviolet optical materials,” SPIE 4842, 259 (2003), http://dx.doi.org/10.1117/12.459479

[2] Frey, B.J., Leviton, D.B., Henry, R.M., Quijada, M.A. "Cryogenic high-accuracy absolute prism refractometer for infrared through far-ultra-violet optical materials: implementation and initial results,” SPIE 5172, 119 (2003), http://dx.doi.org/10.1117/12.506097

[3] Leviton, D.B., Frey, B.J., "Cryogenic, High-Accuracy, Refraction Measuring System - a new facility for cryogenic infrared through far-ultraviolet refractive index measurements,” SPIE 5494, 492 (2004), http://dx.doi.org/10.1117/12.563795

[4] Leviton, D.B., Frey, B.J., Kvamme, T., "High accuracy, absolute, cryogenic refractive index measurements of infrared lens materials for JWST NIRCam using CHARMS,” SPIE 5904, (2005), http://dx.doi.org/10.1117/12.619306

[5] McLean, I.S., et al, "Design and development of MOSFIRE: the multi-object spectrometer for infrared exploration at the Keck Observatory,” SPIE 7735, (2010), http://dx.doi.org/10.1117/12.856715 
[6] Kerber, F., Frey, B.J., Leviton, D.B., Bristow, P., Kaufl, H.U., Pirard, J., Rosa, M.R., "Calibration of the ZnSe pre-disperser on ESO's cryogenic IR echelle spectrograph (CRIRES): comparison of the first results from CRIRES and the laboratory data from CHARMS,” SPIE 6269-, (2006), http://dx.doi.org/10.1117/12.670393

[7] Sharples, R., et al, "Design of the KMOS multi-object integral-field spectrograph,” SPIE 6269, (2006), http://dx.doi.org/10.1117/12.669781

[8] Fabricant, D., et al, " NIRMOS: a wide-field near-infrared spectrograph for the Giant Magellan Telescope," SPIE 8446, (2012), http://dx.doi.org/10.1117/12.926262

[9] Grupp, F., Prieto, E., Geis, N., Bode, A., Katterloher, R., Bodendorf, C., Penka, D., Bender, R., “The EUCLID NISP tolerancing concept and results,” SPIE 9143, (2014), http://dx.doi.org/10.1117/12.2055398

[10] Grupp, F.D., Leviton, D.B., Miller, K.H., Quijada, M.A., “The need for accurate cryo refractive indices and mechanical properties of optical materials (Lithosil 3001, CaF2 and S-FTM16 for EUCLID NISP),” SPIE, San Diego, (2015)

[11] Ricker, G.R., Winn, J.N., Vanderspek, R., Latham, D.W., Bakos, G.A, et al., "Transiting Exoplanet Survey Satellite", J. Astron. Telesc. Instrum. Syst. 1(1), 014003 (Oct 24, 2014), http://dx.doi.org/10.1117/1.JATIS.1.1.014003

[12] Pasquale, B., et al, “Optical Design of the WFIRST-AFTA Wide-Field Instrument,” SPIE 9293, (2014), http://dx.doi.org/10.1117/12.2177847

[13] Groff, T.D., et al, “Construction and status of the CHARIS high contrast imaging spectrograph,” SPIE 9147, 91471W, (2014), http://dx.doi.org/10.1117/12.2055769

[14] Groff, T.D., et al, “The CHARIS IFS for High Contrast Imaging at Subaru,” SPIE, San Diego, (2015)

[15] Leviton, D.B., Frey, B.J., Henry, R.M., “Temperature-dependent refractive index measurements of S-FPL51, S-FTM16, and STIM28 to cryogenic temperatures,” SPIE 8863, (2013), http://dx.doi.org/10.1117/12.2024821

[16] Leviton, D.B., Miller, K.H., Quijada, M.A., Grupp, F.D., “Temperature-dependent refractive index measurements of CaF2, Suprasil 3001, and S-FTM16 for the Euclid Near Infrared Spectrometer and Photometer," SPIE, San Diego, (2015)

[17] Frey, B.J., Leviton, D.B., “Automation, operation, and data analysis in the cryogenic, high accuracy, refraction measuring system (CHARMS),” Proc. SPIE, 5904, 59040P (2005), http://dx.doi.org/10.1117/12.619302

[18] Tropf, W.J., "Temperature-dependent refractive index models for BaF2, CaF2, MgF2, SrF2, LiF, NaF, KCl, ZnS, and ZnSe," Optical Engineering, 34(5), 1369, May 1995, http://dx.doi.org/10.1117/12.201666

[19] Ohara Optical Glass Catalog, http://www.oharacorp.com/ 